\title{
Management of a perio-endo lesion associated with palatogingival groove
}

\author{
Esra Ercan, ${ }^{1}$ Kadir Tolga Ceyhanlı, ${ }^{2}$ Bilge Cansu Uzun, ${ }^{1}$ Buket Kılkış, ${ }^{2}$ Tamer Taşdemir $^{2}$ \\ 'Department of Periodontology, Karadeniz Technical University, Trabzon, Turkey \\ 2Department of Endodontics, Karadeniz Technical University, Trabzon, Turkey
}

The aim of this case report is to present treatment of a lateral incisor with severe bone destruction associated with a palatogingival groove by using endodontic-periodontal multidisciplinary approach. A palatogingival groove (PGG) is a predisposing factor for localized periodontal disease and is rare according to literature reports. It runs to various depths of the root surface of the involved tooth. This report describes the diagnosis and management of a left maxillary lateral incisor with a palatogingival groove extending below the middle third of the root with severe periodontal destruction. The tooth was treated with endodontic therapy and saucerization of the defect surface with resin-modified glass-ionomer cement (RMGIC) after a periodontal flap surgery. There was significantly decreased periradicular radiolucency and, periodontal attachment gain at the 24-month postoperative follow-up.

Keywords: Endodontic treatment; palatogingival groove; periodontal treatment.

$\mathrm{P}$ alatogingival grooves are reported to be seen in the maxillary incisors, predominantly in the lateral incisors. ${ }^{[1]}$ The prevalence is reported to be $2.8-8.5 \%$ of all humans. ${ }^{[2]}$ This anomaly results from infolding of the enamel organ and the Hertwig epithelial sheath in the course of odontogenesis. ${ }^{[3]}$ The groove lies along the palatal surface; thus, it is also known as a distolingual groove or radicular lingual groove. ${ }^{[1]}$ It can occur on the mesial, distal, or facial surface of the tooth and can be unilateral or bilateral. ${ }^{[2]}$

Bacterial invasion from the palatogingival groove, along with a breach of epithelial attachment, can lead to a self-sustaining periodontal defect. Gingival inflammation, plaque accumulation, and deeper probing depth are clear symptoms in these cases. ${ }^{[4]}$ The groove can extend to the various depths and may even communicate with the pulp cavity. ${ }^{[5]}$ Patients cannot completely clean the groove, so periodontal breakdown is near-inevitable. These defects may not be detected until the tooth shows an advanced periodontal defect with pulpal involvement. In this case report, treatment of a left lateral incisor tooth with a deep palatogingival groove on the palatal surface, using combined endodontic and periodontal management was described.

\section{Case report}

A 32-year-old systemically healthy female patient was referred to the Endodontics Department because of a complaint of sinus tract formation on the buccal periapical mucosa of the left lateral incisor (Fig lc). Her family history was non-contributory. She had no periodontal therapy previously. The tooth was asymptomatic with an

Correspondence: Dr. Kadir Tolga Ceyhanlı. Karadeniz Teknik Üniversitesi, Diş Hekimliği Fakültesi, Endodonti Anabilim Dalı, 61080 Trabzon, Turkey.

Tel: +90 462 - 3774775 e-mail: tolgaceyhanli@yahoo.com

Submitted: February 04, 2015 Accepted: May 14, 2015 

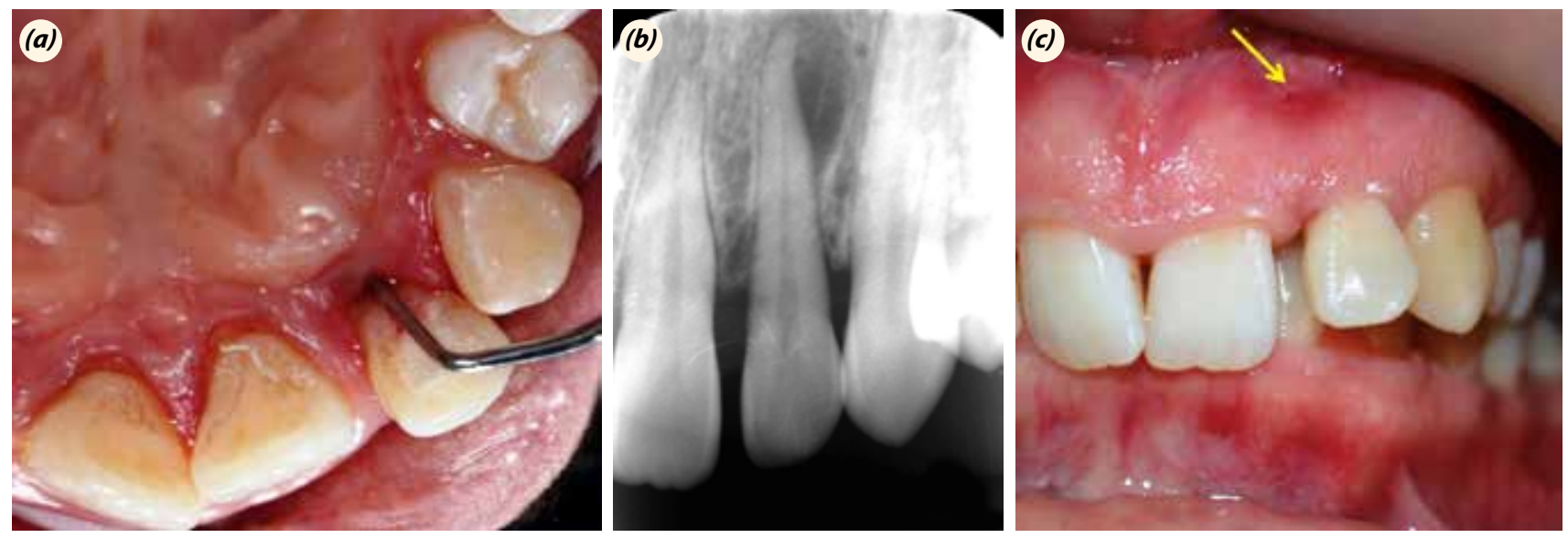

Fig. 1. (a) Preoperative examination with periodontal pocket probing. (b) Radiograph of bony defect surrounding the apical half of the distal side of the left maxillary lateral incisor root. (c) Fistula formation on the buccal mucosa marked with yellow arrow.

intact crown, and had undergone no prior restoration. There was no response to the vitality tests, confirming the diagnosis of a non-vital pulp. The patient noted a mild response to the percussion test.

Localized periodontitis was determined as the diagnosis after a comprehensive periodontal examination. The periodontal pocket probing depth was $6 \mathrm{~mm}$, extending with the palatogingival groove at one point of the midpalate (Fig. la). However, other sides revealed normal probing depths.

There was a large diastema between the central and lateral incisors and the left lateral was unaesthetically positioned in a disto-vestibular position without occlusal interference. However, the patient had no complain about the existing aesthetics and did not want any orthodontic or restorative therapy.
The periapical and periradicular status were examined on a periapical radiograph. There was a radiolucent lesion, revealing a bone defect surrounding the apical half of the distal side of the left maxillary lateral incisor root on the radiographic examination (Fig. lb).

Multidisciplinary treatment was planned, beginning with endodontic treatment of the left lateral incisor. First, an access cavity was prepared under rubber-dam isolation and the canal orifice was explored. The endodontic working length was determined with Root ZX mini apex locator (Morita, USA) and the root canal was prepared with the ProTaper (Dentsply Maillefer, Ballaigues, Switzerland) rotary instrumentation system to size F3. Sodium hypochlorite was used as an irrigation solution between instrumentations. The root canal was dressed with calcium hydroxide for seven days and after removal of the calcium hydroxide, the root canal was filled with $\mathrm{AH}$ plus
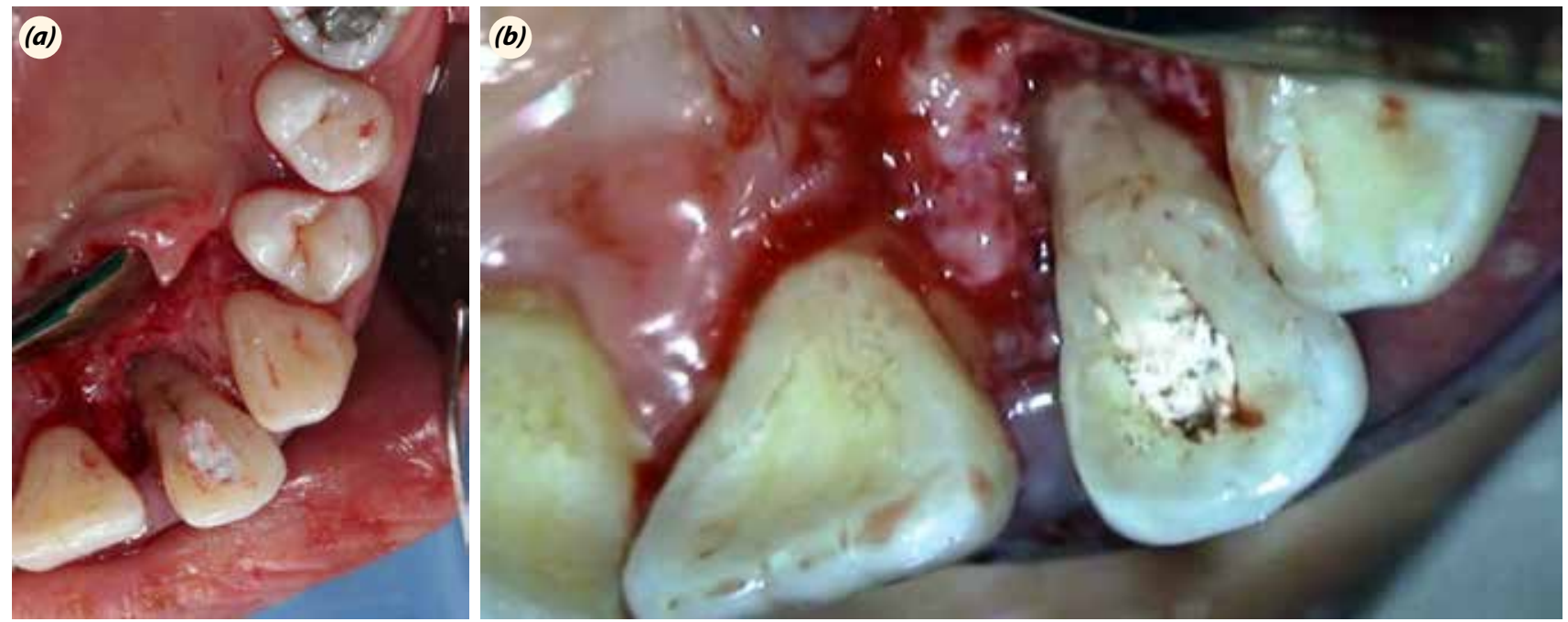

Fig. 2. (a) Appearance of the groove after flap surgery (b) RMGIC restoration under DOM magnification. 

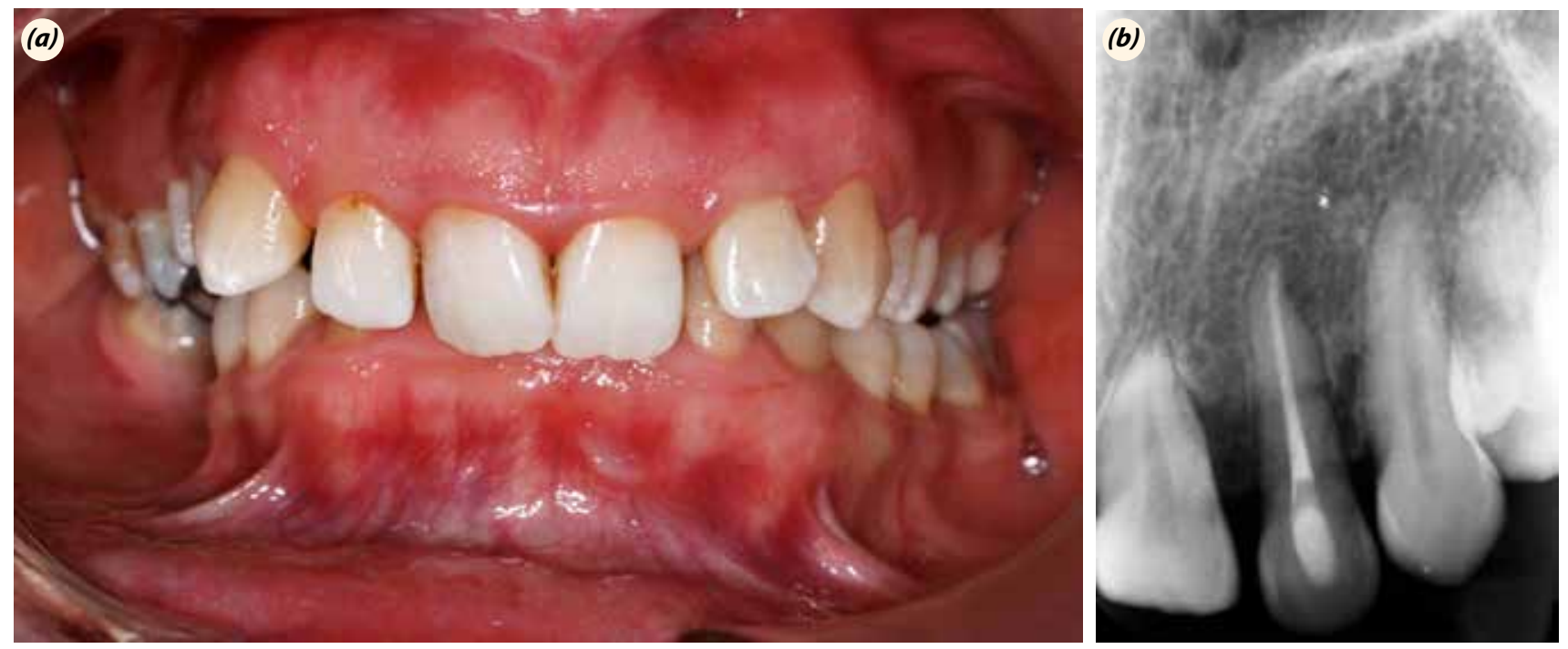

Fig. 3. (a) Clinical and (b) radiographical appearance after 24 months.

(Dentsply DeTrey, Konstanz, Germany) sealer and laterally compacted gutta-percha at the second visit.

The patient was scheduled for periodontal flap surgery after one week. The periodontal flap operation was performed using a dental operating microscope (DOM; Möller Denta 300, HS Möller-Weder, Germany). During the periodontal phase of the therapy, a full-thickness mucoperiosteal flap was reflected on the palatal aspect of the maxillary left lateral incisor. After curettage of granulation tissue, the most apical extent of the palatogingival groove could be clearly observed. The borders of the groove was from the cementoenamel junction to the below of the middle third of the root (Fig. 2a).

Additionally, a horizontal circumferential periodontal defect related to the palatogingival groove was detected below the apical region. Following root planning, odontoplasty of the palatogingival groove defect was performed using a round diamond bur (Mani Inc., Tokyo, Japan) and restored with resin-modified glass-ionomer cement (3M ESPE, USA) under DOM magnification (Fig. 2b). Subsequently, the flap was replaced to its original position and sutured with 3-0 silk sutures (Doğsan, Turkey). One week later, the sutures were removed.

The patient was followed at $1,3,6,12$, and 24 months postoperatively. After 24 months, the tooth was clinically asymptomatic and functional, the probing depth had reduced to three $\mathrm{mm}$, and radiographic healing was evident (Figs. 3a, 3b).

\section{Discussion}

Radicular anomalies, such as a palatogingival groove, create an accessibility problem and act as a "plaque trap".
${ }^{[6,7]}$ With plaque accumulation, these malformations predispose the tooth to severe periodontal disease because of bacterial colonization. Additionally, pulp involvement may occur as a result of the transmission of bacterial toxins between the root canal system and the groove. ${ }^{[3]}$

The prognosis of an affected tooth depends on the extension, location, and depth of the groove. ${ }^{[7]}$ The groove may be shallow sometimes, terminate at the CEJ and may locate in the crown of the tooth. The prognosis is likely good in those cases and requires a simple treatment. Phase 1 periodontal therapy, including scaling and root planning and elimination of the groove, may be considered. On the other hand, if the groove is more advanced, and associated with severe periodontal destruction, the treatment of a palatogingival groove can present a challenge; it may be managed using a multidisciplinary endodontic and periodontal approach. ${ }^{[7]}$ Accessory canals connecting to the pulp in the depth of the grooves can result in bacterial ingress to pulpal tissues; pulpal necrosis may occur as another complication of a palatogingival groove. In our case, the groove extended to the apical third of the root in combination with horizontal supportive bone loss and non-vital pulp. Thus, we decided to treat the patient using a multidisciplinary approach.

Operating microscopes provide homogeneous illumination and a three-dimensional view, which combine to allow clear visualization of the examination site. ${ }^{[8]}$ It is crucial to view the surgical area effectively for a successful treatment. In the present case, the operating microscope provided a detailed view of the root surface with $8 \times$ magnification.

Saucerization, using different materials, such as glass- 
ionomer cement, composite resin, and amalgam, has been advocated for groove restoration. ${ }^{[7]}$ Santos et al. concluded that well-finished resin-modified glass-ionomer cement (RMGIC) and microfill composite (MC) subgingival restorations did not significantly affect periodontal health, and RMGIC may have more positive effects on biofilm composition than MC. ${ }^{[9]}$ Moreover Alkan et al. demonstrated that periodontal health was maintained when RMGIC was used for subgingival or trans-gingival restorations. ${ }^{[10]}$ Thus, RMGIC was used for the restoration of the palatogingival groove in the present case.

\section{Conclusion}

This case report demonstrated healing of the palatogingival groove-related periodontal defect after surgical curettage without placement of a bone graft and with restoration of the groove using RMGIC. In this way, a 3-mm periodontal attachment was gained and periradicular healing was achieved at 24-months.

Conflics of Interest: No conflicts declared.

\section{References}

1. Lara VS, Consolaro A, Bruce RS. Macroscopic and microscopic analysis of the palato-gingival groove. J Endod 2000;26:345-50.

2. Kogon SL. The prevalence, location and conformation of palato-radicular grooves in maxillary incisors. J Periodon- tol 1986;57:231-4.

3. Al-Hezaimi K, Naghshbandi J, Simon JH, Rotstein I. Successful treatment of a radicular groove by intentional replantation and Emdogain therapy: four years followup. Oral Surg Oral Med Oral Pathol Oral Radiol Endod 2009;107:82-5.

4. Withers JA, Brunsvold MA, Killoy WJ, Rahe AJ. The relationship of palato-gingival grooves to localized periodontal disease. J Periodontol 1981;52:41-4.

5. Mayne JR, Martin IG. The palatal radicular groove. Two case reports. Aust Dent J 1990;35:277-81.

6. Storrer CM, Sanchez PL, Romito GA, Pustiglioni FE. Morphometric study of length and grooves of maxillary lateral incisor roots. Arch Oral Biol 2006;51:649-54.

7. Attam K, Tiwary R, Talwar S, Lamba AK. Palatogingival groove: endodontic-periodontal management--case report. J Endod 2010;36:1717-20.

8. Zafersoy-Akarslan Z, Erten H, Uzun O, Semiz M. Reproducibility and agreement of clinical diagnosis of occlusal caries using unaided visual examination and operating microscope. J Can Dent Assoc 2009;75:455.

9. Santos VR, Lucchesi JA, Cortelli SC, Amaral CM, Feres $\mathrm{M}$, Duarte PM. Effects of glass ionomer and microfilled composite subgingival restorations on periodontal tissue and subgingival biofilm: a 6-month evaluation. J Periodontol 2007;78:1522-8.

10. Alkan A, Keskiner I, Yuzbasioglu E. Connective tissue grafting on resin ionomer in localized gingival recession. J Periodontol 2006;77:1446-51. 